\title{
Hallazgos clínicos, bioquímicos y moleculares de la acidemia propiónica. Reporte de un caso
} Clinical, biochemical and molecular findings of propionic acidemia

\author{
Prof. Francisco Cammarata-Scalisia, Dra. Chiu Yen-Hui ${ }^{b}$, Dra. Liu Tze-Tzeb,c, Prof. Gloria Da Silva , \\ Lic. Dianora Araque ${ }^{a}$, Dr. Michele Callea ${ }^{d}$ y Prof. Andrea Avendaño
}

\begin{abstract}
RESUMEN
La acidemia propiónica es un trastorno infrecuente con patrón de herencia autosómico recesivo causado por la deficiencia de la enzima mitocondrial propionil-CoA carboxilasa, que convierte el propionil-CoA a D-metilmalonil-CoA. Se expone el caso de un recién nacido masculino con signos de dificultad respiratoria, vómitos y cansancio durante la alimentación. Presentó acidosis metabólica, cuerpos cetónicos en el suero y la orina positivos, hiperamonemia, anemia, trombocitopenia e hipoproteinemia. El estudio bioquímico por cromatografía de gases acoplada a espectrometría de masas en la muestra de orina fue sugestivo de acidemia propiónica. El estudio molecular en el gen PCCA encontró las mutaciones c.893A>G (p.K298R) en el padre y c.937C $>$ T (p.R313X) en la madre. Existe la necesidad de establecer el diagnóstico de esta entidad infrecuente para implementar las medidas terapéuticas disponibles y aportar el oportuno asesoramiento genético.

Palabras clave: acidemia propiónica, propionil-CoA carboxilasa, gen PCCA, asesoramiento genético.
\end{abstract}

\section{ABSTRACT}

Propionic acidemia is an infrequent disorder with an autosomal recessive inheritance pattern caused by the deficiency of the mitochondrial enzyme propionyl-CoA carboxylase that converts propionyl-CoA to D-methylmalonylCoA. We present the case of a male newborn who showed signs of respiratory distress, vomiting and tiredness during feeding. He presented metabolic acidosis, positive serum and urine ketone bodies, hyperammonemia, anemia, thrombocytopenia and hypoproteinemia. The biochemical study by gas chromatography coupled to mass spectrometry in a urine sample was suggestive of propionic acidemia.

a. Unidad de Genética Médica, Departamento de Puericultura y Pediatría, Universidad de Los Andes, Mérida, Venezuela.

b. Department of Education and Research, Taipei City Hospital, Taipei, Taiwan.

c. VYM Genome Research Center, National Yang-Ming University, Taipei, Taiwan.

d. Unit of Dentistry, Bambino Gesù Children's Hospital, IRCSS, Rome, Italy.

\section{Correspondencia:}

Prof. Francisco Cammarata-Scalisi:

francocammarata19@gmail.com

Financiamiento: Ninguno.

Conflicto de intereses: Ninguno que declarar.

Recibido: 28-7-2018

Aceptado: 2-1-2019
The molecular study in the PCCA gene found the mutations c.893A>G (p.K298R) in the father and c.937C> T (p.R313X) in the mother. There is a need to establish the diagnosis of this infrequent entity to implement the therapeutic measures available and provide the appropriate genetic counseling. Key words: propionic acidemia, propionyl-CoA carboxylase, PCCA gene, genetic counseling.

http: / / dx.doi.org/10.5546/ aap.2019.e288

Cómo citar: Cammarata-Scalisi F, Yen-Hui C, Tze-Tze L, Da Silva G, et al. Hallazgos clínicos, bioquímicos y moleculares de la acidemia propiónica. Reporte de un caso. Arch Argent Pediatr 2019;117(3):e288-e291.

\section{INTRODUCCIÓN}

La acidemia propiónica (AP, OMIM \#606054) es una entidad metabólica hereditaria de los ácidos orgánicos que presenta un patrón de herencia autosómico recesivo, causado por la deficiencia de la enzima mitocondrial propionilCoA carboxilasa (PCC), la cual está compuesta de dos subunidades, una $\alpha$ (PCCA, OMIM \#232000), cuyo gen se localiza en 13q32, y una $\beta$ (PCCB, OMIM \#232050), localizada en 6p21. Esta enzima es dependiente de biotina y cataliza la carboxilación de propionil-CoA a D-metilmalonilCoA, que se encuentra involucrada en el catabolismo de los aminoácidos de cadena ramificada, ácidos grasos de cadena impar, ${ }^{1-4}$ cadenas laterales del colesterol, timina y uracilo, ${ }^{1}$ y fermentación bacteriana de los carbohidratos en el intestino. ${ }^{4} \mathrm{La}$ incidencia mundial estimada es de 1 en 50 000-100 000 recién nacidos. No obstante, puede presentarse desde 1 en 1000 en Groenlandia hasta 1 en 660000 en Taiwán. ${ }^{5}$

Alrededor del $80 \%$ de los pacientes presenta la forma de aparición temprana o neonatal aguda (diagnosticada antes de los tres meses de edad). Corresponde la forma clásica y más grave, caracterizada por presentar síntomas clínicos sugestivos de intolerancia a las proteínas, como descompensación grave, determinada por acidosis metabólica e hiperamonemia, además de letargia, vómitos, rechazo al alimento, deshidratación, hipotonía, convulsiones y arritmias cardíacas, 
que pueden causar la muerte a edades tempranas. La segunda forma de aparición es la tardía o progresiva crónica, la cual muestra retardo en el crecimiento y del desarrollo. ${ }^{3,6-9}$ Esta entidad se asocia, por lo general, con complicaciones neurológicas; $;^{2,3,7,8}$ además, puede presentar miocardiopatía, ${ }^{2,8}$ mayormente dilatada. Se ha asociado con el síndrome de QT largo, ${ }^{2}$ alteraciones gastrointestinales, renales, hematológicas, inmunológicas, dermatológicas ${ }^{3,10}$ y trastorno del espectro autista. ${ }^{11}$

Se expone el caso de un recién nacido con hallazgos clínicos y bioquímicos de AP para dar a conocer la forma de presentación más común y, así, facilitar la sospecha diagnóstica y el rápido reconocimiento por parte de neonatólogos y pediatras.

\section{CASO CLÍNICO}

Recién nacido masculino, producto de padres sanos y no consanguíneos. La madre era segunda gesta, con un aborto previo no estudiado. El embarazo fue controlado, complicado con infección de las vías urinarias al final de la gestación. Nació por cesárea segmentaria a las 38 semanas, sin complicación. El peso al nacer fue de $3000 \mathrm{~g}$, con puntaje $\mathrm{z}-0,6$, y la talla, de $51 \mathrm{~cm}$, con puntaje $\mathrm{z} 0,7$.

Al tercer día de nacido, presentó signos de dificultad respiratoria y decaimiento, sin fiebre ni cianosis. Fue hospitalizado con los diagnósticos iniciales de sepsis y deshidratación. Al momento del examen físico de ingreso, se evidenció mucosa oral seca, frecuencia respiratoria de 90 respiraciones por minuto, aleteo nasal, tiraje intercostal y subcostal. Ante la auscultación, murmullo vesicular rudo con roncus bilaterales, y el resto de la exploración, dentro de la normalidad.

Los cultivos de líquido cefalorraquídeo, sangre, orina y heces no arrojaron la presencia de agentes patógenos. El perfil de toxoplasmosis, rubeola, citomegalovirus, herpes simple y otros agentes (toxoplasmosis, rubella, cytomegalovirus, herpes simplex virus and other agents; TORCH, por sus siglas en inglés) fue igualmente negativo. Las transaminasas hepáticas y el perfil tiroideo se encontraron dentro de la normalidad. Presentó elevación en las cifras de lactato deshidrogenasa, cuerpos cetónicos en el suero y la orina positivos, hipocalcemia e hiperfosfatemia. Se evidenció hiperamonemia (con valores que oscilaron entre 208 y $1470 \mu \mathrm{g} / \mathrm{dl}$ ), que fue tratada con cese del aporte proteico, alto aporte calórico y carnitina.
No pudieron usarse quelantes de amonio ante la sospecha de acidemia orgánica y hemodiálisis por el bajo peso del paciente. Presentó, además, episodios de hipo- e hiperglucemia. A los 14 días de nacido, sufrió hipoproteinemia con disminución de las cifras de albúmina e hiperbilirrubinemia a expensas de la indirecta. Permaneció hospitalizado en la Unidad de Cuidados Intensivos Neonatales, sin evidenciar evolución clínica satisfactoria hasta los 32 días de nacido.

La ecografía transfontanelar realizada a los seis días de nacido fue normal y la renal mostró doble sistema pielocalicial izquierdo. La evaluación cardiovascular evidenció insuficiencia aórtica leve y displasia tricúspide con regurgitación moderada.

Luego de una leve mejoría a los 53 días, presentó vómito de contenido alimentario en dos oportunidades. Concomitantemente, disminución en la succión y cansancio durante la alimentación, por lo que fue hospitalizado de nuevo. La hematología completa evidenció anemia grave de $3,5 \mathrm{~g} / \mathrm{dl}$ y plaquetopenia de $17000 \mathrm{~mm}^{3}$, por lo cual recibió transfusiones sanguíneas en dos oportunidades, sin presentar mejoría. Se encontró, además, en acidosis metabólica por estudios de gases arteriales. El estudio bioquímico por cromatografía de gases acoplada a espectrometría de masas en la muestra de orina mostró excreción aumentada de 2-hidroxibutirato, 3-hidroxipropionato, 3-hidroxibutirato, propionilglicina PG1 y PG2, metilcitrato, 3-hidroxivalerato, tigliglicina TG1 y TG2 y glicina. Ante estos hallazgos, se inició el uso de fórmula indicada para la AP.

A los 59 de días, presentó cianosis, convulsión tónico clónica, por lo que se ingresó a la Unidad de Cuidados Intensivos Pediátricos y recibió respiración asistida. Se evidenció una leve mejoría, con medidas nutricionales y fórmula láctea específica para AP. Sin embargo, se mantuvo la hiperamonemia con cifras de $289 \mu \mathrm{g} /$ dl y, a los dos meses y 20 días, presentó un paro cardiorrespiratorio, el cual no respondió a las maniobras de reanimación.

Posteriormente al fallecimiento del paciente, se dispuso del estudio molecular por secuenciación de Sanger en los genes PCCA y PCCB en los progenitores, para confirmar su estado de portador, y se encontraron las mutaciones c.893A > G (p.K298R) en el padre y c.937C > T (p.R313X) en la madre, ambas en el gen PCCA (Figura 1). 


\section{DISCUSIÓN}

La AP es causada por la baja actividad de la PCC, que produce la generación tóxica del ácido propiónico a partir de la propionil-CoA acumulado en la matriz de las mitocondrias. ${ }^{8,9}$ Este defecto genera la acumulación de intermediarios metabólicos, como el ácido 3-hidroxipropiónico y metilcitrato en la orina y la propionilcarnitina en la sangre. La hiperamonemia descrita puede ser debida a la inhibición del ciclo de la urea, que contribuye a la morbimortalidad. ${ }^{6}$

La acumulación de ácidos orgánicos, transportada a través de la barrera hematoencefálica o sintetizada en el sistema nervioso central, conduce a las alteraciones metabólicas debido a su toxicidad. Esto causa daño cerebral e inhibición del metabolismo mitocondrial, que explica la acidosis, la hipoglucemia y la hiperamonemia. Además, un déficit secundario de carnitina genera la disminución en la energía y el estrés oxidativo se debe a la disminución de la capacidad antioxidante. $^{3}$

En la actualidad, no existe tratamiento curativo para la AP, por lo que el manejo es sintomático, con dieta baja en proteínas de alta energía o el suplemento con mezclas específicas libres de sustratos propiogénicos o precursores del propionil CoA, como los aminoácidos de cadena ramificada (valina, isoleucina, metionina, treonina) y ácidos grasos de cadena impar, ${ }^{6,8}$ con el objeto de minimizar la producción de metabolitos tóxicos. ${ }^{5}$ Se debe aumentar la ingesta de carbohidratos, evitar largos períodos de ayuno, suplir con L-carnitina para eliminar el exceso de ácido propiónico, ${ }^{4,5,7}$ emplear laxantes para tratar o prevenir la constipación y antibióticos (metronidazol) para prevenir el sobrecrecimiento bacteriano. ${ }^{4} \mathrm{El}$ trasplante de hígado se ha usado para mejorar los síntomas de la hiperamonemia, ${ }^{7}$ la supervivencia, la calidad de vida, para prevenir el deterioro neurológico ${ }^{12}$ y para la reversión de la cardiomiopatía. ${ }^{13}$ Sin embargo, estos solo mejoran parcialmente los síntomas y el resultado general en la forma grave sigue siendo decepcionante. Por lo tanto, existe la necesidad de desarrollar un tratamiento alternativo duradero y seguro. ${ }^{9}$

Entre las complicaciones presentes en el trasplante de hígado en dos centros europeos, la mortalidad estuvo en el $58 \%$ con empeoramiento de la función renal. No obstante, se recomienda monitorear las funciones cardíacas y renales antes y después de la intervención, usando inmunosupresión renal. Se sospecha que algunas de las complicaciones pueden estar relacionadas con la toxicidad acumulada por el defecto. ${ }^{14}$

Después del fallecimiento del paciente, y ante el seguimiento en los progenitores, se dispuso y se realizó un estudio molecular en ellos y se encontró una nueva mutación tipo cambio de sentido en el gen PCCA en el padre, el cual resultó patogénica según el programa PolyPhen-2. Por su parte, la madre presentó una mutación sin sentido en el mismo gen, por lo que se podría intuir que el paciente estudiado fue heterocigoto compuesto. Las mutaciones en el gen PCCA fueron las más frecuentes en una serie de 25 pacientes, pues se encontró en 18 de ellos. La mutación encontrada en la madre estuvo presente en 9 alelos (cuatro pacientes como homocigotos y uno como heterocigoto compuesto), asociada a la aparición temprana de la entidad y a la muerte antes del año de edad. ${ }^{15}$
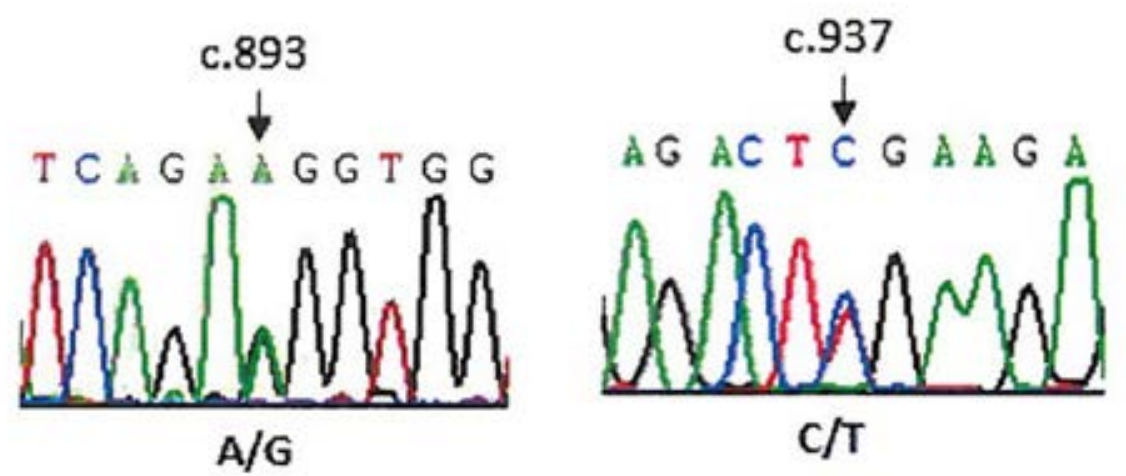
En conclusión, después del descarte de infección y otras alteraciones comunes en el período neonatal e infantil que pueden llevar, en ocasiones, a una evolución tórpida, se debe plantear la presencia de un error innato del metabolismo. El diagnóstico clínico en la AP se puede confirmar por estudios bioquímicos y el análisis molecular del gen PCCA o PCCB. Por lo general, estos estudios son de difícil acceso en nuestra región y pueden retrasar el diagnóstico. Ante los hallazgos clínicos y de laboratorio presentes de forma precoz en el niño, se solicitó el estudio bioquímico para establecer el diagnóstico de AP. Además, se identificó el estado de portador sano en sus progenitores. A pesar del manejo sintomático brindado al paciente, la evolución fue desfavorable. Ante la presencia de una entidad con patrón de herencia autosómico recesivo, se debe brindar un oportuno asesoramiento genético familiar con riesgo de recurrencia del $25 \%$ para futuras gestaciones de la pareja.

\section{Agradecimientos}

Al Doctor Emanuele Bellacchio, Research Laboratories, Bambino Gesù Children's Hospital, IRCCS, Rome, Italy.

A la Licenciada Rosalía Gumina F., directora de la Biblioteca del Instituto Autónomo Hospital Universitario de Los Andes, Universidad de Los Andes.

\section{REFERENCIAS}

1. Grünert SC, Bodi I, Odening KE. Possible mechanisms for sensorineural hearing loss and deafness in patients with propionic acidemia. Orphanet J Rare Dis. 2017; 12(1):30.

2. Fuertes Moure A, Centeno Jiménez M, Álvarez GarcíaRovés R, Gil Villanueva N, et al. Acidemis propiónica y síndrome QT largo: una asociación potencialmente grave. An Pediatr (Barc). 2015; 83(4):281-2.
3. Arias C, Raimann E, Peredo P, Cabello J, et al. Propionic acidemia and optic neuropathy: a report of two cases. JIMD Rep. 2014; 12:1-4.

4. Daly A, Pinto A, Evans S, Almeida MF, et al. Dietary practices in propionic acidemia: A European survey. Mol Genet Metab Rep. 2017; 13:83-9.

5. Chiu YH, Liu YN, Liao WL, Chang YC, et al. Two frequent mutations associated with the classic form of propionic acidemia in Taiwan. Biochem Genet. 2014; 52(9-10):415-29.

6. Chapman KA, Collado MS, Figler RA, Hoang SA, et al. Recapitulation of metabolic defects in a model of propionic acidemia using patient-derived primary hepatocytes. Mol Genet Metab. 2016; 117(3):355-62.

7. Rafique M. Emerging trends in management of propionic acidemia. Arq Bras Endocrinol Metabol. 2014; 58(3):237-42.

8. Gotoh K, Nakajima Y, Tajima G, Watanabe Y, et al. Determination of methylmalonyl coenzyme A by ultra high-performance liquid chromatography tandem mass spectrometry for measuring propionyl coenzyme A carboxylase activity in patients with propionic academia. J Chromatogr B Analyt Technol Biomed Life Sci. 2017; 1046:195-9.

9. Darvish-Damavandi M, Ho HK, Kang TS. Towards the development of an enzyme replacement therapy for the metabolic disorder propionic acidemia. Mol Genet Metab Rep. 2016; 8:51-60.

10. Grünert SC, Müllerleile S, De Silva L, Barth M, et al. Propionic acidemia: clinical course and outcome in 55 pediatric and adolescent patients. Orphanet J Rare Dis. 2013; 8:6.

11. Witters P, Debbold E, Crivelly K, Vande Kerckhove K, et al. Autism in patients with propionic acidemia. Mol Genet Metab. 2016; 119(4):317-21.

12. Li M, Dick A, Montenovo M, Horslen S, et al. Costeffectiveness of liver transplantation in methylmalonic and propionic acidemias. Liver Transpl. 2015; 21(9):1208-18.

13. Arrizza C, De Gottardi A, Foglia E, Baumgartner M, et al. Reversal of cardiomyopathy in propionic acidemia after liver transplantation: a 10-year follow-up. Transpl Int. 2015; 28(12):1447-50.

14. Charbit-Henrion F, Lacaille F, McKiernan P, Girard M, et al. Early and late complications after liver transplantation for propionic acidemia in children: a two centers study. Am J Transplant. 2015; 15(3):786-91.

15. Gupta D, Bijarnia-Mahay S, Kohli S, Saxena R, et al. Seventeen novel mutations in PCCA and PCCB genes in Indian propionic acidemia patients, and their outcomes. Genet Test Mol Biomarkers. 2016; 20(7):373-82. 